\title{
Desafíos de la educación secundaria en Argentina: Pensar juntos algunas transformaciones necesarias y posibles*
}

\section{Challenges of Secondary Education in Argentine: Thinking together some necessary and possible transformations}

\author{
Horacio Ademar Ferreyra
}

\section{Resumen}

A partir del conocimiento construido en un estudio acerca de las características y procesos de la Educación Secundaria en Argentina en el período 2000-2010, desarrollado con el propósito de caracterizar los principales retos y posibilidades de este tramo del sistema educativo, se presentan en este artículo algunas propuestas de cambio que implican transformaciones necesarias y posibles con la intención de aportar a la resignificación del nivel. Como alternativa a un enfoque jerárquico de arriba-abajo o abajo-arriba, que sitúa en la parte superior a los gobiernos y en la base a quienes trabajan en las escuelas, se propugnan otras lógicas y dinámicas para estas relaciones. Desde ellas, se enuncian algunas acciones prioritarias para las políticas educativas en los ámbitos escolar (micro), provincial (meso) y federal (macro), con especial atención a sus interacciones. La idea central es la necesidad de una real apropiación de los cambios concertados en el nivel federal por parte de los sujetos (personas e instituciones) que dan vida a la educación: las jurisdicciones y las escuelas.Palavras-chave: Gestão compartilhada, participação, relação escola e comunidade.

Palabras Clave: Cambio organizacional, educación secundaria, política educacional, política gubernamental, ciencias de la educación

Abstract

Parting from the knowledge acquired in a study on characteristics and processes of Secondary Education in Argentine, within the period of time 2000-2010, carried out in order to identify the main challenges and possibilities of this part of the education system; this article presents some proposals for change, which involve the necessary and possible transformations, to contribute to reorganization of this education level, as an alternative to a hierarchic approach up-bottom or bottom-up, which places governments in the upper part, and those working at school in the bottom, other logic and dynamics are proposed for these relationships. From such relationships, some priority actions are included for education policies at school, (micro), provincial, (meso), and federal, (macro), with special attention to their interactions. The central idea is the need of a truly implementation of changes agreed at federal level by the subjects, (individuals and institutions), which are the reason of education existence: jurisdiction and schools.

Keywords: Organization change, secondary education, education policy, government policy, education sciences

*Este artículo recupera y actualiza desarrollos construidos por el Grupo de Estudio de Educación Secundaria (Facultad de Educación, Universidad Católica de Córdoba- Argentina) en el marco del proyecto de investigación que se propuso caracterizar la Educación Secundaria argentina en el período 2000-2010, para considerarla en relación con sus principales retos y posibilidades. Los resultados de dicho estudio han quedado sistematizados en Ferreyra, H. (coord.) (2012). Entramados, análisis y propuestas para el debate. Aproximaciones a la Educación Secundaria en la Argentina (2000-2010). Córdoba, Argentina: Comunic-Arte-UCC [CD Rom]. 


\section{Introducción}

En el proceso de cambio educativo, pensar y hacer educación desde una mirada constructiva supone abarcar el legado de saberes, prácticas y creencias acumulados en el pasado (para revalorizarlo y también para interpelarlo), interpretar los signos de los nuevos escenarios con apertura a las innovaciones del presente y vislumbrar y edificar los mejores futuros posibles. Esta es la perspectiva que, en nuestra investigación sobre Educación Secundaria en Argentina, nos ha impulsado a ir más allá de la caracterización y contextualización de este trayecto educativo en la primera década del siglo XXI en Argentina, para asumir una prospectiva de cambio, con la convicción de que la calidad es posible a partir de la generación de condiciones de equidad en las trayectorias educativas, de dispositivos institucionales situados e innovadores, de mejores climas institucionales, de experiencias de aprendizaje más ricas para los jóvenes de la escuela secundaria, de acuerdos entre actores educativos y sociales, de políticas - provinciales y nacionales- que sostengan y orienten el cambio.

La intención es aportar a los procesos de resignificación de la Educación Secundaria, a partir del reconocimiento de los aspectos que debieran modificarse (por su ya probada ineficacia), los que demandan una revisión en virtud de los nuevos requerimientos de la época y aquellos que debieran tener continuidad, fortaleciéndose, porque dan sentidos a la función educativa en cada una de las escalas/territorios que configuran el sistema educativo nacional. Hablamos de re-significar porque entendemos que es necesario desentrañar los sentidos implícitos en los lenguajes, relatos y gramáticas de la escuela secundaria para crear nuevas narrativas que den indicio o signo de una realidad distinta.

El punto de partida de las proyecciones que se repasan en este artículo es la idea de que la re-significación de la Educación Secundaria, en tanto sistema nacional, depende, en gran medida, de una real apropiación de los cambios concertados en el nivel federal por parte de los sujetos (personas e instituciones) que dan vida a la educación: las jurisdicciones y las escuelas. Correlativamente, propugnamos otras lógicas para estas relaciones, de modo que, como alternativa a un enfoque jerárquico de arriba-abajo o a la inversa, que sitúa en la parte superior a los gobiernos y en la base a quienes trabajan en las escuelas, sea posible concebir un conjunto de círculos concéntricos en cuyo núcleo se ubiquen los estudiantes y sus familias en interacción con sus profesores en la escuela, y desde donde las relaciones se vayan extendiendo hasta abarcar la comunidad, la región/zona/distrito, la provincia/jurisdicción y el Estado Nacional.

A la hora de considerar los diversos factores influyentes en la Educación Secundaria, una representación como la que estamos proponiendo nos permite trabajar con una imagen de relaciones simultáneas que circulan de adentro hacia afuera y de afuera hacia adentro (Darling-Hammond, 2001), o bien como sostiene Martinic (2001)- de relaciones que se construyen hacia adentro y afuera. Así, las interacciones y las influencias son multidireccionales: todos y cada uno de los componentes conforma y es conformado por los demás. Como argumenta Darling-Hammond (2001): "No es plausible una visión dura de la reforma emprendida desde arriba, ni tampoco otra romántica, dejada al albur de los cambios espontáneos desde las bases. Son necesarios tanto la imaginación local como el liderazgo político" (p. 274). Este es el marco desde el cual enunciamos, a continuación, algunas orientaciones que podrían contribuir a la re-significación deseada.

\section{Las políticas educativas en el ámbito escolar (micropolítica)}

En este ámbito la gestión debiera tener como meta la promoción de la calidad educativa, procurando las condiciones necesarias para la inclusión, el reconocimiento, la integración y el logro educativo de todos los jóvenes en la educación obligatoria. Esto supone considerar a la escuela como una organización, como un sistema escolar (micro) que forma parte de un sistema educativo (meso/macro); como una institución en situación, perfectible, inteligente, sensible, democrática, ética, dinámica, constructiva y emprendedora; con capacidad de crecer a partir de un plan o proyecto común (con los encuentros y desencuentros inherentes) centrado en la enseñanza y el aprendizaje en contexto. Estimamos 
que este sería el campo propicio para que en cada escuela secundaria considerada como una unidad pedagógica y organizativa- se tomaran decisiones, de manera democrática y participativa, de acuerdo con los lineamientos nacionales y provinciales vigentes, efectuando al mismo tiempo planteamientos propios acordes con la historia, la cultura y el contexto de cada institución, en un movimiento que pone en relación lo global y lo local.

Un plan de acción abierto y flexible (Plan Educativo Institucional -PEI-), compuesto por actividades sistemáticas, coordinadas, integrales e integradas facilitaría la visión del todo, sin perder de vista la centralidad de lo pedagógico como marco de referencia para la acción. Es en este proyecto donde se deberían integrar todos los programas y proyectos que, tanto desde la jurisdicción como de la instancia nacional, llegan a las escuelas, a los fines de fortalecer la enseñanza para potenciar los aprendizajes de todos los estudiantes. Se evitaría de este modo la proliferación desarticulada que supone la suma de proyectos que fragmenta la gestión en vez de integrarla e impide que las innovaciones sucedan.

Es imprescindible que la tarea educativa de cada institución se centre en sus estudiantes y en el logro de más y mejores aprendizajes, lo cual significa pensar las escuelas con capacidad para identificar necesidades y articular respuestas acordes que al reconocer, valorar y desarrollar las potencialidades de todos y cada uno de los estudiantes, garanticen el derecho a aprender. Solo de esta manera es posible alcanzar la calidad con pertinencia, relevancia, eficiencia, eficacia y equidad (Orealc/Unesco, 2007; Blanco, et al 2008). En este contexto, se requiere una revisión reflexiva de las prácticas docentes en vistas a la generación de cambios en la cultura institucional que posibiliten el abordaje de los problemas como oportunidades para profundizar los procesos de inclusión. Es este también el ámbito donde podrían generarse y sostenerse los tiempos y espacios necesarios para que los educadores potencien su desarrollo profesional centrando en la escuela (Vezub, 2009; Lombardi y Vollmer, 2010).

Asimismo, constituye un imperativo de la gestión escolar habilitar canales de participación y concertación para que todos los sujetos involucrados en el proceso formativo (directivos, docentes, estudiantes, exalumnos, familias, representantes de las organizaciones de la sociedad civil, empresarios, etc.) asuman su papel de protagonistas comprometidos y no de meros trasmisores o espectadores (Blejmar, 2005; Ferreyra y Peretti, 2006; Ferreyra, 2009; Romero, 2009; Bolívar, 2009). Solo de este modo es posible construir en redes una comunidad educativa que dé vida y busque el fortalecimiento intra e interinstitucional. Entendemos que es en esta línea donde se inscribe el movimiento de "buenas escuelas", "escuelas inclusivas", o sea, de "escuelas que trabajan bien", dispuestas a modificar las prácticas de gestión y concentrar esfuerzos en la calidad de la educación y la promoción humana, generando cambios que se traducen en innovaciones hacia adentro y hacia fuera en función de la proximidad con los participantes, los requerimientos de la comunidad de pertenencia y en el marco del sistema educativo.

\section{Las políticas educativas en el ámbito provincial (mesopolítica)}

En este ámbito, el desafío es doble: sostener y profundizar los procesos de participación en el Consejo Federal de Educación (CFE) ${ }^{1} \mathrm{y}$, al mismo tiempo, pasar del énfasis puesto en el conocimiento de las prescripciones contenidas en los acuerdos emanados de este organismo a su efectiva apropiación, mediante un trabajo sistemático con los equipos técnicos, supervisores, directivos y docentes de aula. El proceso podría comenzar, teniendo en cuenta el grado de implementación que cada jurisdicción ha alcanzado respecto de los distintos acuerdos, por redefinir el sentido y las funciones de la Educación Secundaria en virtud de su obligatoridad (Ciclo Básico y Orientado) o, en su defecto, por revisar el proceso de modificaciones en marcha teniendo en cuenta su multidimensionalidad, dando participación no solo a los docentes, sino a otros actores familias, estudiantes y comunidad con el propósito de identificar las fortalezas y debilidades de

1. En Argentina, el Consejo Federal de Educación (CFE) es el organismo de concertación, acuerdo y coordinación de la política educativa nacional para asegurar la unidad y articulación del Sistema Educativo Nacional. Su presidente es el Ministro de Educación de la Nación y se encuentra integrado por la máxima autoridad educativa de cada jurisdicción y tres representantes del Consejo de Universidades. 
las acciones desarrolladas para propiciar las mejoras correspondientes, re-significando y reorientando la política educativa. Esto supone asumir la necesaria e inevitable situacionalidad de la política educativa en cada contexto provincial en el concierto nacional.

Aquellas jurisdicciones que a la fecha no cuentan con Ley Provincial de Educación sancionada con posterioridad a la Ley Nacional de Educación necesitan adecuar la regulación del sistema local a partir de la concertación con los distintos actores de la comunidad educativa. Las que ya poseen leyes o normativas específicas podrán avanzar con su reglamentación a los fines de garantizar el derecho a una educación de calidad a todos los jóvenes. Es necesario que las modificaciones que resulten de la implementación de los cambios se regulen mediante normas explícitas, construyendo de esta manera un plexo normativo actualizado.

En lo que respecta a la implementación del Ciclo Básico y Orientado, aquellas jurisdicciones que aún no la han realizado podrían asumir la construcción de un mapa de localización de la oferta y estudiar las cuestiones referidas a reubicación del personal docente en el nivel local a través de un trabajo colaborativo entre los equipos técnicos provinciales y las escuelas. En cambio, las que se encuentran avanzadas en la definición de la cartografía deberían detenerse a evaluarla, con el fin de efectuar los ajustes que permitan generar más y mejores oportunidades educativas para todos los jóvenes en sus respectivos territorios. En este sentido, resulta imprescindible pensar en cómo abordar la secundaria en ámbitos rurales y en cómo atender a la población que presenta sobreedad, entre otros imperativos.

Consideramos imprescindible definir en las jurisdicciones una política de articulación intra e inter niveles y/o ciclos, particularmente con la Educación Primaria y de manera especial con la Educación Superior y el sistema científico tecnológico. Esto supone asumir la articulación como una cuestión institucional más allá de la mera relación de contenidos entre grados o cursos-, como una estrategia que permita complementar los esfuerzos, optimizar el uso de los recursos y garantizar el tránsito de los estudiantes por los distintos niveles, ciclos y trayectos.
A las jurisdicciones que aún no han iniciado el proceso de construcción curricular o que lo tienen en marcha, les queda por delante el desafío de afrontarlo desde un modelo participativo, que no solo involucre a los docentes, directivos, supervisores y técnicos como protagonistas centrales del hecho educativo, sino a la sociedad en su conjunto. Podrían tener en cuenta -contextualizándolos en sus propias realidades los dispositivos y dinámicas que siguieron aquellas provincias que presentan avances en este sentido.

Por otra parte, consideramos que los nuevos diseños y/o propuestas curriculares, antes de su aprobación definitiva, deberían validarse en la práctica durante un año como mínimo para facilitar los procesos de apropiación y ajuste que median entre el proyecto y su desarrollo. A su vez, las jurisdicciones que elaboraron sus diseños y propuestas curriculares antes de la aprobación de los acuerdos federales (Núcleos de Aprendizajes Prioritarios ${ }^{2}$ para el Ciclo Orientado y Marcos de Referencia ${ }^{3}$ para cada una de las Orientaciones) necesitan concentrar sus esfuerzos en la revisión, a los fines de efectuar, de ser necesarias, las modificaciones que se consideren pertinentes y relevantes en cada contexto, a partir de la validación que se haya podido realizar en la práctica.

En todos los casos, resulta de vital importancia pensar en diseños y desarrollos dinámicos, que pongan su acento en la enseñanza, que se monitoreen de manera permanente y que cada cuatro o cinco años se revisen integralmente y se modifiquen formalmente, instalando un continuum de accióninvestigación-reflexión que potencie los aprendizajes y el diálogo constructivo entre todos los sujetos que interactúan: Escuela-Provincia ${ }^{4}$. De esta manera, para 2. En la República Argentina, desde el Consejo Federal de Educación, se ha acordado la aprobación de los Núcleos de Aprendizajes Prioritarios (NAP) con la intención de promover la integración del Sistema Educativo Nacional. Los NAP para los diferentes Niveles y Ciclos fueron elaborados por representantes políticos, técnicos, supervisores y docentes de las provincias argentinas y de la Ciudad de Buenos Aires y por los equipos técnicos del Ministerio de Educación nacional, y aprobados por las autoridades educativas de todas las Jurisdicciones.

3. Los Marcos de Referencia para la Educación Secundaria Orientada constituyen la manifestación del acuerdo federal acerca de la formación específica para cada una de las Orientaciones: Ciencias Sociales/Ciencias Sociales y Humanidades, Ciencias Naturales, Economía y Administración, Lenguas, Agrario/Agro y Ambiente, Comunicación, Informática, Educación Física, Turismo, Arte (con diferentes énfasis: Música, Teatro, Danza, Artes Visuales, Diseño, Artes Audiovisuales, Multimedia u otras).

4. Podría revisarse la experiencia desarrollada en la provincia de Córdoba respecto del proceso de validación y aprobación de diseños curriculares por 
actualizar los distintos dispositivos que involucran a las escuelas, la Provincia y la Nación, se ingresa en un proceso permanente y dinámico de construcción y reconstrucción curricular.

En cada contexto es imprescindible el análisis de las normativas que regulan las prácticas de enseñanza y aprendizaje (regímenes de evaluación, promoción y acreditación, ingreso, equivalencias, certificaciones y títulos) a fin de adecuarlas a las necesidades y particularidades provinciales en el marco de la política federal. Asimismo, las jurisdicciones deben trabajar para adecuar los regímenes laborales docentes a las exigencias actuales, para hacerlos más consistentes con los propósitos de calidad, igualdad, inclusión, gestión y participación deseados. Por ejemplo, a través de la instrumentación de mecanismos que posibiliten la concentración horaria en pocos establecimientos, la evaluación de la labor docente (Marcelo, 2009), la aplicación de incentivos por el desempeño o la localización laboral en áreas vulnerables, la determinación de los perfiles docentes para cada una de las disciplinas, materias o espacios curriculares de los nuevos diseños y/o propuestas curriculares; mecanismos de ingreso, promoción y acceso por medio de concursos de antecedentes $\mathrm{y} / \mathrm{u}$ oposición; establecimiento de tiempos específicos (intensivos o extensivos) para el abordaje colectivo de las temáticas y acciones institucionales (en el nivel de cursos, ciclos, etc.), entre otros temas.

Resulta prioritario potenciar el uso de la información cuantitativa y cualitativa producida de manera conjunta entre el Ministerio Nacional y las jurisdicciones con los fines de alimentar los procesos de toma de decisiones en el nivel provincial y de las escuelas. Para ello, deberá potenciarse el trabajo articulado en torno a la evaluación: de aprendizajes, docente e institucional.

Otra tarea decisiva es fortalecer los sistemas provinciales de planificación educativa, mediante el desarrollo de herramientas conceptuales y metodológicas que permitan diseñar y poner en marcha un sistema de información y comunicación democrático, sistemático y confiable entre el Ministerio (Plan de Desarrollo Educativo Provincial), las Unidades de Supervisión (Plan de Supervisión un período determinado (5 años).
Regional) y las Escuelas (Plan Educativo Institucional) en el marco del Plan Nacional de Educación. A ello, sumamos la necesidad de generar los mecanismos de enlace y coordinación de los diferentes planes, programas, proyectos y acciones pedagógicas, curriculares, comunitarias y administrativas que se desarrollan en las distintas escalas/territorios del sistema educativo.

En todas las provincias, se debería poner mayor énfasis en la profundización de aquellas acciones que posibiliten más autonomía pedagógica y organizativa de las instituciones educativas, que articulen las regulaciones vigentes con las particularidades locales e institucionales a fin de establecer -en el marco de sus respectivos PEI y a partir de procesos de autoevaluación permanente- planes anuales de mejora orientados a superar problemas o situaciones que preocupan por su gravedad o por su urgencia, con el objetivo de no dispersar esfuerzos humanos, funcionales, materiales y económicos. En esta línea, es necesario evaluar las experiencias que se están desarrollando en distintas instituciones en el marco de los planes de mejora de la Educación Secundaria.

La implementación de las nuevas propuestas formativas requiere de acciones de formación docente continua situadas que respondan a los requerimientos provinciales y escolares. En este sentido, y a los fines de acompañar y potenciar los procesos de cambio puestos en marcha, se han de potenciar los dispositivos centrados en la escuela y la enseñanza, propiciar el trabajo en redes escolares, desarrollar ciclos de formación destinados a profesores y directivos para apoyar los nuevos roles y funciones, implementar postgrados y/o postítulos, entre otras posibilidades, con el propósito de fortalecer la gestión del cambio en cada una de las escuelas de las jurisdicciones.

En esta línea, es prioritario centrar esfuerzos en la articulación del Plan de Desarrollo Educativo Provincial con las propuestas de formación continua establecidas en el Programa Nacional de Formación Permanente (Resolución CFE $N^{o}$ 201/13) en el marco de Plan Nacional de Educación Obligatoria y Formación Docente 2012 - 2016 (Resolución CFE 188/12), y las prioridades de las escuelas. 
Todas estas acciones deberán estar acompañadas de una mayor inversión en educación, lo que supone establecer claramente cuál es la prioridad que se le da a la educación en el proyecto de país para, en función de ello, asignarle las partidas presupuestarias (en su escala macro y meso) que hagan posible potenciar la construcción de una Educación Secundaria de calidad para todos los jóvenes de cada una de las provincias y, por ende, de la Argentina en el siglo XXI.

\section{Las políticas educativas en el nivel federal (macropolítica)}

En este ámbito es fundamental continuar potenciando y jerarquizando el funcionamiento del CFE como organismo interprovinciales, de carácter permanente, como ámbito de concertación, acuerdo y coordinación de la política educativa, asegurando la unidad y la articulación del sistema. Esto requiere afianzar las acciones iniciadas para potenciar la participación de los actores nacionales, fuerzas políticas, sociales, religiosas y productivas, entre otras, en la construcción de un Proyecto Educativo Nacional que se constituya en el núcleo central de una política de Estado para el desarrollo económico, social, político y cultural de nuestro país ${ }^{5}$.

En este sentido, también se debiera fortalecer la Mesa Federal de Secretarios/Subsecretarios de Educación de las Jurisdicciones, coordinada por la Subsecretaría de Equidad y Calidad, así como las mesas de trabajo por niveles educativos y modalidades y los encuentros intersectoriales, para potenciar al máximo la capacidad de planeamiento e implementación de acciones educativas. Esta acción participativa, en el caso específico de la Educación Secundaria, tendría que estar acompañada por diversas instancias de evaluación sistemática de los acuerdos federales y programas que orientan y apoyan los procesos de toma de decisión, con la intencionalidad de abordar no solo las cuestiones emergentes (fenoménicas), sino también aquellos componentes o aspectos estructurales que facilitan $\mathrm{y} / \mathrm{u}$ obstaculizan dichos procesos o acciones iniciados en cada una de las 5. Para ello, se deberían fortalecer los mecanismos previstos en la Ley de Educación Nacional para el acompañamiento de los procesos de toma de decisiones -Consejo de Políticas Educativas, Consejo Económico y Social y Consejo de Actualización Curricular- con el objeto de permitir la participación de todos los que, de una u otra manera, están vinculados con la problemática de la Educación Secundaria. jurisdicciones, desde una perspectiva nacional pero a su vez contextualizada en las particularidades regionales.

$\mathrm{Al}$ respecto, y teniendo en cuenta los resultados de la investigación realizada, aparecen como prioritarias las siguientes líneas de acción:

- Acordar nuevas metas en el tiempo para la implementación de los distintos ciclos que integran la Educación Secundaria en virtud de los avances que evidencia cada una de las jurisdicciones;

- consolidar las acciones de planificación y evaluación de la educación en el marco del Plan Nacional de Educación Obligatoria y Formación Docente 2012-2016, considerando las metas de corto, mediano y largo plazo;

- evaluar los Planes de Mejora Provinciales de Educación Secundaria y con base en ello proyectar los planes para el período 2014-2016 y, en este marco, continuar con el financiamiento de Planes de Mejora Institucional, mediante la asignación de recursos a las escuelas para que lleven a cabo las acciones previstas en ellos ${ }^{6}$ :

- continuar con el Programa Conectar Igualdad Modelo 1 a 1 (Lugo y Kelly, 2011) y potenciar el uso pedagógico de las TIC en el aula, las escuelas y la comunidad;

- profundizar los procesos de homologación de títulos de la Secundaria Orientada y los referentes a la movilidad estudiantil;

- dar continuidad al acompañamiento técnico y financiero a las provincias en los procesos de implementación de los Acuerdos Federales mediante los Planes de Apoyo a la Educación Inicial, Obligatoria y Modalidades.

Además, será necesario re-pensar las políticas socioeducativas para el Nivel Secundario y Modalidades a partir del desarrollo de acciones auténticas de discriminación positiva, con el fin de contribuir a la disminución de las desigualdades, 6. Se considera prioritario fortalecer la construcción de la línea de base como herramienta que potencia el diseño y gestión de las políticas, incluyendo no solo información cuantitativa sino también cualitativa que oriente los procesos de toma de decisión. 
la fragmentación social y los circuitos educativos segmentados en las distintas jurisdicciones. Estas acciones involucran lo social -en un sentido amplio-, interpelan fuertemente al contexto territorial, a la comunidad y a todas las instituciones que aportan al fortalecimiento de los procesos educativos. La mejora de las condiciones y posibilidades en el acceso, la calidad en el itinerario escolar y los resultados alcanzados (en términos de apropiación de los saberes necesarios para la vida social, cultural, política, económica y comunitaria) constituyen el horizonte de las políticas socioeducativas. Es por ello que se deberían retomar y reorientar los diferentes programas, estrategias y acciones existentes para afrontar los desafíos actuales que plantea la política educativa en el campo de la Educación Secundaria y Modalidades. Las políticas socioeducativas debieran fundamentarse en un concepto amplio de inclusión, que entiende que es necesario garantizar el derecho al acceso y es fundamental trabajar por una inclusión educativa con calidad, donde todos puedan ejercer el derecho a la educación.

En este sentido, creemos que es necesario focalizar algunas acciones en aquellas instituciones a las que asisten sujetos en situación de vulnerabilidad social y educativa, pero también universalizar otras, como así también centralizar o descentralizar las acciones que se consideren necesarias en virtud de la realidad, a los fines de contribuir con el proceso de inclusión educativa en términos de igualdad de condiciones y calidad de los procesos y resultados.

Dichas políticas deberían diseñarse y gestionarse desde una perspectiva integral (sectorial) pero a la vez integrada (intersectorial) con otras políticas de promoción personal, social y de la ciudadanía que sostienen el Estado Nacional, las jurisdicciones y las organizaciones de la sociedad civil. Esto supone seguir apostando a una construcción de políticas desde una perspectiva relacional, que apunte a integrar los términos de las tensiones clásicas (propias de las últimas décadas del Siglo XX) -centralización $v s$ descentralización, universalidad $v s$ focalización, sectorial vs intersectorial, entre otras- en la búsqueda de puntos de equilibrio en la generación de políticas sociales y educativas.
En este marco, se podrían incluir entre otras líneas de acción, las siguientes:

- Ampliar y afianzar el actual sistema de becas para los estudiantes de Educación Técnica, incorporando a los de algunas de las Orientaciones de la Secundaria que se consideren también prioritarias para el desarrollo sustentable en cada provincia y, por ende, en el nivel nacional;

- incorporar becas estudiantiles cuyo otorgamiento no esté solo asociado a condiciones de vulnerabilidad socioeconómica, sino también al esfuerzo y rendimiento de los estudiantes ${ }^{7}$;

- continuar con las becas para estudiantes de pueblos originarios, padrinazgo presidencial, hijos de ex combatientes de Malvinas y para erradicación del trabajo infantil;

- dar continuidad a la línea de provisión de libros (de texto, literatura y consulta) y adquisición de útiles, de manera descentralizada, en el marco de un proceso que contemple la participación de las escuelas en la selección de los bienes mencionados, sobre la base de sus respectivos planes educativos institucionales y las prioridades establecidas por cada jurisdicción y la Nación;

- fortalecer y expandir el Proyecto para la Prevención del Abandono Escolar;

- dar continuidad a los Centros de Actividades Juveniles diversificando sus actividades a los fines de ampliar los horizontes culturales de los jóvenes, y afianzando su articulación e integración en los respectivos Planes Educativos Institucionales;

- continuar apoyando propuestas socioeducativas que apunten a garantizar la Educación Secundaria;

- ampliar - previa evaluación- los Programas Turismo Educativo y Recreativo, Orquestas y Coros, entre otros.

7.Se sugiere revisar la experiencia de Becas Provinciales que está desarrollando la provincia de Córdoba para estudiantes del Ciclo Orientado o Superior de la Modalidad Técnico Profesional (Véase Programa Provincial de Becas Estudiantiles -Nivel Secundario- en http://www.igualdadycalidadcba. gov.ar/SIPEC-CBA/areas/subdireccion/socioeducativas/socioeducativas4. $\underline{\mathrm{html}}$ ). 
En otro orden, y con el propósito de garantizar los estudios secundarios, deberían evaluarse a los fines de generar conocimiento las experiencias que se están desarrollando en distintas jurisdicciones ${ }^{8}$ como alternativas posibles para que los jóvenes con sobreedad que en algún momento interrumpieron estudios puedan retomarlos, y quienes no han podido acceder, los inicien. En este mismo sentido, se debería comenzar a pensar en estrategias posibles para acercar la Educación Secundaria en sus dos ciclos a las poblaciones rurales o alejadas de los centros urbanos. Para ello, resultaría conveniente vincular la Modalidad de Educación Rural a los lineamientos estratégicos acordados federalmente, estudiar las experiencias en marcha ${ }^{9}$ y las acciones concretadas en el marco del Programa de Mejoramiento de la Educación Rural (Promer), entre otras iniciativas, a los fines de generar propuestas que permitan atender la diversidad de situaciones que se presentan en el territorio nacional y, de esta manera, asegurar a los jóvenes del ámbito rural oportunidades de acceso a una Educación Secundaria de calidad.

En lo que respecta a las políticas pedagógicas y curriculares, aparecen como necesarias, entre otras, las siguientes acciones:

- Concretar la aprobación de los marcos de referencia para las nuevas Orientaciones de la Educación Secundaria incorporadas por Resolución 210/13 del CFE (Letras, Físico Matemática y Pedagógica), así como la revisión y ampliación de las Orientaciones Agraria / Agro y Ambiente y Turismo, tal como lo establece la referida resolución.

- Revisar la configuración de la Secundaria Orientada en Arte, en particular los énfásis (lenguajes).

- Evaluar la apropiación de los NAP del Ciclo Básico y de Formación General del Ciclo Orientado en las jurisdicciones.

- Avanzar en las discusiones respecto de los regímenes académicos de la Educación Secundaria;

8. Ciudad de Buenos Aires (Escuelas de Reingreso), Provincia de Buenos Aires (Centros de Escolarización Secundaria para Adolescentes y Jóvenes), Córdoba (Programa de Inclusión para la Terminalidad de la Educación Secundaria y Formación Laboral para Jóvenes de 14 a 17 años), entre otras. En este sentido, podrían consultarse los trabajos realizados en la Línea Educar en Ciudades que promueven la OEI y EuroSocial.

9. Por ejemplo, en las provincias de Córdoba, Buenos Aires, Entre Ríos, entre otras.
- establecer un sistema federal de monitoreo de coherencia curricular que permita efectuar un seguimiento de las decisiones que en cada jurisdicción se van adoptando, a los fines de lograr unidad en la diversidad; asesorar a las provincias en la elaboración de sus respectivos diseños curriculares.

Con referencia a la formación docente, es necesario impulsar todas aquellas políticas que tiendan a jerarquizar, profesionalizar y mejorar las condiciones materiales y culturales de los docentes. Para ello, el sistema formador deberá no solo atender la formación inicial, sino también abordar la formación continua en el marco de las políticas de desarrollo profesional docente. En este sentido, se debería afianzar una mayor articulación entre el Instituto Nacional de Formación Docente y las políticas de cada uno de los niveles y modalidades del sistema educativo.

Respecto de la Formación Inicial, se debería continuar debatiendo en profundidad dónde, cómo, en qué y con qué recursos se formarán los docentes. En este sentido, se impone continuar incentivando la apertura -en los institutos superiores de formación docente y en las universidades- de carreras (profesorados) cuyos títulos habilitan para el desempeño de la docencia en aquellos campos (materias y/o espacios curriculares) que más lo requieran, en virtud de que la demanda supera ampliamente la oferta. Además, a los fines de dar respuesta a un importante grupo de técnicos superiores y profesionales que se han incorporado (ante la ausencia de perfiles con títulos docente) o desean incorporarse como profesores, se debería ampliar la oferta de ciclos o trayectos de formación pedagógica para este tipo de agentes, como complemento de la formación epistemológica y en disciplinas que ya poseen, a fin de que los titulados en distintas especialidades puedan obtener una certificación o título que valide sus competencias profesionales docentes ${ }^{10}$.

Respecto de la Formación Continua, y tal como ya se ha enfatizado en el nivel de las jurisdicciones, se debe afianzar el desarrollo de una política que incluya, entre otras acciones, una definición conjunta, por parte del Ministerio Nacional y de las provincias,

10. Las Universidades, por lo general, se han hecho cargo de la formación docente de los campos no tradicionales (Comunicación Social, Ciencias Políticas, etc.). 
de planes, programas y/o proyectos de formación para los docentes en ejercicio en el marco de los Planes de Apoyo a la Educación Inicial, Obligatoria y Modalidades.

Dichas acciones deberán contemplar las exigencias, necesidades y prioridades del sistema educativo que provienen de las instituciones, de los proyectos o de las características de comunidades educativas particulares y de las inquietudes personales de los docentes en un área o problemática socioeducativa determinada. Esta construcción -que ya viene desarrollándose- entre la Nación y cada provincia, debería perfeccionarse a los fines de contemplar las particularidades regionales y respetar los ritmos de implementación de la transformación en cada uno de los contextos provinciales, en el marco de los Acuerdos Federales.

En esta línea, aparece como un claro desafío la puesta en marcha del Programa Nacional de Formación Permanente 2013-2016, que se propone articular:

La jerarquización de la Formación Docente y la calidad de los aprendizajes, articulando procesos de formación con mecanismos de evaluación y fortalecimiento de la unidad escuela; como ámbito privilegiado de desempeño laboral y a la vez espacio de participación, intercambio y pertenencia. (Anexo Resolución CFE N 201/13, p. 2).

Constituye un reto relevante el cumplimiento efectivo de la intención de atender, a la vez, la integralidad del sistema educativo nacional y la construcción de los consensos necesarios con los gobiernos educativos de las jurisdicciones en relación con las prioridades de formación.

La premisa es que el desarrollo profesional de los docentes en ejercicio (maestros, profesores, directores, supervisores, tutores de curso, coordinadores o jefes de departamentos) supone un trabajo que debe favorecer la autonomía y la participación, profundizar el acrecentamiento de sus aprendizajes, capacidades y competencias profesionales a partir de un trabajo in situ (en la escuela), con estilo colaborativo (no solo individual), abierto al aporte de saberes externos, el intercambio de experiencias y la reflexión colectiva sobre lo hechos y las cuestiones pendientes, a los fines de contribuir con su desarrollo personal, social y profesional en contexto. En este marco, será necesario revisar las acciones desarrolladas y potenciar el desarrollo profesional a través de otras estrategias -más allá del curso tradicional-, como por ejemplo: capacitación centrada en la escuela ${ }^{11}$, participación en redes de formación e intercambio ${ }^{12}$, ciclos de formación ${ }^{13}$, postgrados y postítulos.

En el marco de la formación, también se debería seguir propiciando un trabajo articulado entre las jurisdicciones y los Institutos de Formación Docente y Universidades para el desarrollo de actividades ligadas a la investigación de temáticas referidas a la enseñanza, el trabajo docente y la formación docente; al asesoramiento pedagógico a las escuelas, a la preparación para el desempeño de cargos directivos y de supervisión, al acompañamiento de los primeros desempeños profesionales, a la formación para el cumplimiento de distintas funciones, a la producción de materiales para la enseñanza, entre otras posibilidades.

Corresponde al Ministerio Nacional continuar potenciando el desarrollo de estrategias integrales de evaluación de la calidad y el uso integral de la información que circula en el sistema, articulando los dispositivos nacionales con los provinciales (Relevamientos anuales, operativos nacionales y provinciales de evaluación de la calidad, informes mensuales, cédulas escolares ${ }^{14}$, entre otros). Pensar

11. Asesoramiento pedagógico a las escuelas, elaboración y desarrollo de Proyectos Curriculares e Institucionales, grupos de innovación en y entre las escuelas, ateneos pedagógicos para la discusión de casos, documentación de experiencias pedagógicas, seminarios de profundización teórica, pasantías en otras instituciones, apoyo profesional mutuo entre colegas y con la colaboración de expertos, tutorías de un docente experimentado con amplia trayectoria, entre otros.

12.Mesas redondas, jornadas de presentación y discusión de experiencias, talleres o seminarios con especialistas sobre un tema de interés de los docentes de la red, publicación de materiales didácticos, secuencias de enseñanza y recursos elaborados por la docentes de la red; boletín electrónico con noticias de interés para la comunidad docente, eventos y reseñas bibliográficas, foros electrónicos para el debate sobre temas que preocupan a los docentes, entre otros.

13.Análisis de casos, estudios de incidentes críticos y propuesta de cursos alternativos de acción, lectura y discusión de bibliografía, conferencias y paneles a cargo de expertos, presentaciones, explicaciones y desarrollos a cargo de formadores; elaboración de planes de trabajo y proyectos a cargo de los participantes, entre otros.

14.Se sugiere recuperar la experiencia que está desarrollando la Dirección General de Cultura y Educación de la Provincia de Buenos Aires (véase http://abc.gov.ar/escuelas/consultas/cedulaescolar/default.cfm; fecha de 
en una evaluación auténtica supone diseñarla tomando como punto de partida el derecho de todos a aprender; en este sentido, no se trata de establecer estándares como parámetros de control de estudiantes, docentes y escuelas ni como mecanismos para clasificar instituciones educativas, sino de fijar unas metas a alcanzar -currículum común- y valorar su grado de consecución en los centros educativos, en contexto, atendiendo a la variabilidad y particularidad de cada escuela (Darling Hammond, 2001; Bolívar, 2003a y b). Esta estrategia de "establecer estándares sin estandarizar" concierne, como sostiene Bolívar (2003a):

A asegurar una equidad en la educación (...) el foco se cambia ahora de la cantidad a la calidad, del control al apoyo, del rendimiento burocrático a la responsabilidad pública (a la comunidad) por el trabajo desarrollado. De este modo, las decisiones tomadas con motivo de la evaluación pueden tener mejor potencial para mejorar la calidad de los aprendizajes (p. 8)

Por otra parte, es fundamental continuar apoyando el desarrollo de estrategias de trabajo conjunto entre Nación, jurisdicción y escuelas con el fin de que los sujetos involucrados se apropien de los dispositivos de evaluación de los aprendizajes y a partir de sus resultados puedan generar acciones que potencien la mejora. Además, posibilitar el desarrollo de investigaciones de los distintos programas y proyectos, como así también la socialización de experiencias innovadoras con el propósito de reorientar los procesos de generación de políticas de mejora en las distintas escalas/territorios que conforman el sistema educativo nacional.

De lo que se trata, en definitiva, es de consolidar un Ministerio de Educación Nacional con alta capacidad profesional, dinámico y a la vez eficiente, que a partir del respeto de las particularidades regionales apoye las necesidades de los equipos técnico-políticos de las distintas jurisdicciones para hacer realidad la centralidad de las políticas de inclusión, justicia e integración; pedagógicas y curriculares, de docencia e investigación, instaurando una visión que, superando

consulta: 25/02/2012) y la microexperiencia puesta en marcha por el Ministerio de Educación de la Provincia de Córdoba en el año 2002-2003 (véase http://ieo.wikispaces.com/file/view/Modelo+de+Cedula+Escolar.pdf ). los acotados límites de los tiempos políticos, proyecte y actúe en los amplios tiempos de la educación.

En esta etapa de re-significación de la Educación Secundaria el desafío es doble. Por un lado, seguir profundizando el papel del Estado en el diseño y gestión de Política Educativa desde una perspectiva relacional tendiente a generar condiciones de igualdad para todos los adolescentes y jóvenes del país, lo que supone -entre otras cuestiones- consolidar los procesos de concertación y participación social tomando decisiones para el sector que tiendan a dar unidad al sistema educativo nacional, respetando el federalismo y las condiciones particulares de cada una de las jurisdicciones y de sus escuelas. Por otro, concentrar esfuerzos y acciones orientados a que los cambios concertados en el nivel federal puedan ser realmente apropiados por los sujetos (personas e instituciones) que dan vida a la educación: las jurisdicciones y las escuelas.

\section{Conclusiones}

En Educación -decimos con Terigi (2005): "Vamos aprendiendo a lo largo de la historia, en el curso de las prácticas..." (p. 66), aprendiendo con y de otros, compartiendo dudas y hallazgos, abiertos a la escucha y al diálogo, con el empeño y las expectativas siempre puestos en la innovación educativa, en la escuela y en los derechos de los estudiantes. Se trata, entonces, de sumar oportunidades para seguir pensando, investigando y aprendiendo con otros, en un intento de conocer más para contribuir a la posibilidad de hacer mejor.

Re-significar la Educación Secundaria desde esa nueva lógica de círculos concéntricos cuyo núcleo son los estudiantes requiere de la articulación de políticas que refuercen y sostengan la centralidad de escuela atendiendo a su sentido, su contexto, su crecimiento y mejora:

- En cuanto al sentido: escuelas donde se enseñe y se aprenda implementando diversidad de estrategias, utilizando intensivamente variados recursos didácticos, contextualizando las propuestas formativas sin perder de vista los aprendizajes considerados 
prioritarios o imprescindibles. Escuelas donde los adultos de la comunidad escolar ubiquen el ingreso, reingreso, permanencia, progreso y egreso de todos los estudiantes en el centro de sus preocupaciones y ocupaciones; donde se confíe en que todos los adolescentes y jóvenes pueden aprender, se alienten y fortalezcan sus logros, se actúe institucionalmente en la búsqueda de alternativas superadoras de sus dificultades (precariedad socioeconómica, problemáticas familiares o personales, privación cultural, entre otras posibles).

- En cuanto al contexto: escuelas donde se trabaje intensamente en un clima cooperativo y solidario, en un ambiente con identidad, donde se valoren las producciones de estudiantes y docentes; escuelas en las que el espacio institucional -material y simbólico"haga lugar" a los mensajes que se quieren transmitir, a las obras que se desea compartir, a las situaciones que son importantes para la comunidad educativa.

- En cuanto al crecimiento y la mejora: escuelas en las que las capacidades profesionales se acrecienten a partir de la práctica cotidiana cooperativa, el aporte de saberes externos, la reflexión sobre lo construido y sobre los desafíos pendientes. Escuelas donde se acumule e integre el "capital pedagógico y didáctico" de la experiencia de todos los docentes, de modo que se pueda ampliar el repertorio de propuestas de enseñanza que permitan atender la diversidad de los estudiantes. Escuelas inclusivas, comprometidas, dispuestas a dar y recibir aportes de su entorno.

En definitiva, la escuela secundaria deberá asumir a la educación como una práctica social emancipadora que "cataliza la realidad socio-educativa", con la intencionalidad de que las personas aprendan a comunicarse, trabajar y participar como ciudadanos (con derechos y obligaciones) y no como meros habitantes en la sociedad actual. Esto supone vivir la escuela en un proceso permanente de aprendizaje situado.

Dejamos así planteados algunos desafíos de la Educación Secundaria en Argentina, convencidos de que las transformaciones necesarias y posibles son tareas de la sociedad en su conjunto. Los adolescentes y jóvenes argentinos esperan respuestas que les permitan no solo conocer, sino ser, hacer y vivir mejor. Garantizarles el derecho a la educación ofrece la oportunidad de hacer posible la realización de todos los otros derechos humanos.

\section{Referencias bibliográficas}

Blanco, R; Aguerrondo, I; Calvo, G; Cares, G; Cariola, L; Cervini, R...Zorilla, M. (2008). Eficacia escolar y factores asociados en América Latina y el Caribe. Santiago: Orealc/Unesco Santiago. Recuperado de http://unesdoc. unesco.org/images/0016/001631/163174s. pdf.

Blejmar, B. (2005). Gestionar es hacer...que las cosas sucedan. Buenos Aires: Noveduc.

Bolívar, A. (2003a). Si quiere mejorar las escuelas, preocúpese por capacitarlas: el papel del rendimiento de cuentas por estándares en la mejora. En Profesorado, Revista de Currículum y Formación del Profesorado, V.7, $N^{o} 1$ - 2, 79-94. Recuperado de http:// redalyc.uaemex.mx/pdf/567/56770205.pdf.

-. (2003 b). Evaluación de centros: ¿Estándares para una mejora interna? En Moreno, J.M. (coord.) Organización y gestión de centros educativos. Madrid: UNED.

(2009). La gestión integrada e interactiva. En Romero, Cl. (comp.). Claves para mejorar la Escuela Secundaria (pp. 41-59). Buenos Aires: Novedades Educativas.

Darling-Hammond, L. (2001). El derecho de aprender. Crear buenas escuelas para todos. Barcelona: Ariel.

Ferreyra, H. y Peretti, G. (comps.) (2006). Diseño y gestión de una Educación Auténtica. Buenos Aires: Novedades Educativas.

Ferreyra, H; Cingolani, M., Eberle, M., Ferreyra, H., Gallo, G., Larrovere, C., Luque, M., Pasut, M., Peretti, G. y Rimondino, R. (2009). Educación Secundaria Argentina. 
Propuestas para superar el diagnóstico y avanzar hacia su transformación. Buenos Aires: Ediciones Novedades Educativas y Academia Nacional de Educación.

Ferreyra, H. (2012). Entramados, análisis y propuestas para el debate. Aproximaciones a la Educación Secundaria en la Argentina (2000-2010). Córdoba, Argentina: Comunic-Arte-UCC [CD Rom].

Lombardi, G. y Vollmer, M.I. (2010). La formación docente como sistema: de la formación inicial al desarrollo profesional. Reflexiones a partir de la experiencia argentina. En Vélaz de Medrano, C. y Vaillant, D. (coords.) Aprendizaje y Desarrollo profesional docente (pp.59-66). Madrid: OEI .Fundación Santillana.

Lugo, M. T y Kelly, V (2011). El modelo 1 a 1: un compromiso por la calidad y la igualdad educativas. La gestión de las TIC en la escuela secundaria: nuevos formatos institucionales. Buenos Aires: Ministerio de Educación de la Nación.

Marcelo, C. (2009). La evaluación del desarrollo profesional docente: de la cantidad a la calidad. En Revista Brasileira de Formação De Professores. 1 (1): 43-70.
Martinic, S. (2001). Conflictos políticos e interacciones comunicativas en las reformas educativas en América Latina. En Revista Iberoamericana de Educación, $\mathrm{N}^{\circ}$ 27, 18-19.

Orealc/Unesco (2007). El derecho a una educación de calidad para todos en América Latina y el Caribe. En REICE - Revista Electrónica Iberoamericana sobre Calidad, Eficacia y Cambio en Educación. 5( 3): 1-21.

Romero, C. (2009). Claves para mejorar la escuela secundaria. La gestión, la enseñanza y los nuevos actores. Buenos Aires: Novedades Educativas.

Terigi, F. (2005). Collected papers: sobre las decisiones en el gobierno de la educación. En Frigerio, G. y Diker, G. (comps.) Educar: ese acto político. Buenos Aires: Del Estante.

Vezub, L. (2009). El desarrollo profesional docente centrado en la escuela. Concepciones, politicas y experiencias. Buenos Aires: Iipe Unesco. 